\title{
The entrainment theorem for wall driven boundary layer flows
}

\section{Journal Article}

Author(s):

Magyari, E.

Publication date:

2008-12

Permanent link:

https://doi.org/10.3929/ethz-b-000069157

Rights / license:

In Copyright - Non-Commercial Use Permitted

Originally published in:

Acta Mechanica 201(1-4), https://doi.org/10.1007/s00707-008-0077-8 


\title{
E. Magyari
}

\section{The entrainment theorem for wall driven boundary layer flows}

\author{
Dedicated to Professor Wilhelm Schneider on the occasion of his 70th birthday
}

Received: 11 September 2007 / Revised: 24 December 2007 / Published online: 12 September 2008

(c) Springer-Verlag 2008

\begin{abstract}
Boundary layer flows driven by permeable plane surfaces, stretching with power-law velocities are considered in the presence of an applied lateral mass flux. The relationship between the wall shear stress and the entrainment velocity (the transversal velocity at the outer edge of the boundary layer) as a function of the mass transfer parameter $f_{\mathrm{w}}$ is examined analytically by using the Merkin transformation method. It is shown that at the value of $f_{\mathrm{w}}$ where the wall shear stress vanishes, the entrainment velocity reaches a minimum or maximum value. This relationship between two characteristic quantities at the outer and inner edge of the boundary layer, respectively, is referred to as entrainment theorem. Its physical content is analyzed in the paper in some detail.
\end{abstract}

\section{Introduction}

Flows driven by moving boundaries belong to the classical issues of fluid mechanics. Stokes' celebrated first and second problem are the oldest examples of such flows. The well-known Couette flows also belong to this class of phenomena.

In addition to these classical cases, in some modern mechanical manufacturing and forming processes, as, e.g., during the drawing of plastic sheets by extrusion of a molten material through a narrow slot, the glass-fiber and paper production, the melt spinning, the cooling of large metallic plates in a bath, etc., in the stagnant ambient fluid a further important class of wall driven flows with boundary layer aspects (far downstream from the slot) arises. Usually, the steady regimes of such processes are of practical relevance.

The first investigation of the steady boundary layer flows induced by continuous stretching surfaces was conducted by Sakiadis [1]. Since the pioneering work of Sakiadis, the theory of these wall driven flows has been much generalized and refined (see, e.g., [2]). Following the papers of [3-8] and of many other authors, the first comprehensive mathematical investigation of the similar "Sakiadis flows" has been reported by Banks [9].

The present paper revisits the problem of the (forward and backward) boundary layer flows induced by permeable plane surfaces stretching with power-law velocities [9], in the presence of an applied lateral mass flux [10]. Its focus is a specific physical and mathematical feature of these flows, namely on the relationship between the wall shear stress and the entrainment velocity as a function of the mass transfer parameter (suction/injection parameter). The main result of the paper is summarized in a short statement referred to as entrainment theorem, which asserts that at the value of the mass transfer parameter where the wall shear stress vanishes, the entrainment velocity of the flow reaches a minimum or maximum value. The converse statement of the entrainment theorem is also true. Its physical content is analyzed in this paper in some detail. 


\section{Basic equations and problem formulation}

The steady boundary layer flow induced by a permeable plane surface stretching with velocity $U_{\mathrm{w}}(x)$ in a quiescent incompressible fluid is governed by equations $[9,10]$,

$$
\begin{aligned}
& \frac{\partial u}{\partial x}+\frac{\partial v}{\partial y}=0, \\
& u \frac{\partial u}{\partial x}+v \frac{\partial u}{\partial y}=v \frac{\partial^{2} u}{\partial y^{2}}
\end{aligned}
$$

subject to the boundary conditions

$$
u(x, 0)=U_{\mathrm{w}}(x), \quad v(x, 0)=V_{\mathrm{w}}(x), \quad u(x, \infty)=0 .
$$

The positive $x$-axis points in the direction of motion of the surface issuing from the slot ( $z$-axis). The $y$-axis is perpendicular to $x$ and to the direction of the slot. $u$ and $v$ are the $x$ and $y$ components of the velocity field, respectively, and $v$ denotes the kinematic viscosity of the fluid. It is further assumed that the stretching velocity has the power-law form

$$
U_{\mathrm{w}}(x)=U_{0} \cdot\left(\frac{x}{L}\right)^{m}
$$

where $-\infty<m<+\infty$ and $-\infty<U_{0}<+\infty$. The reference length $L$ denotes the distance $x$ from the slot where the stretching velocity has the prescribed value $U_{0}$, i.e., $U_{\mathrm{w}}(L)=U_{0}$. Concerning the variation of $U_{\mathrm{w}}(x) / U_{0}$ with increasing values of $x$ in the range $x>L$, the following stretching regimes as functions of the power-law exponent $m$ can be distinguished:

$$
\begin{array}{ll}
m<-1 & : \text { rapidly decreasing stretching velocity } U_{\mathrm{w}}(x) / U_{0}, \\
m=-1 & : \text { inverse linearly decreasing stretching velocity } U_{\mathrm{w}}(x) / U_{0}, \\
-1<m<0 & : \text { slowly decreasing stretching velocity } U_{\mathrm{w}}(x) / U_{0}, \\
m=0 & : \text { uniformly moving surface, } U_{\mathrm{w}}(x) / U_{0}=1, \\
0<m<1 & : \text { slowly increasing stretching velocity } U_{\mathrm{w}}(x) / U_{0} \\
m=+1 & : \text { linearly increasing stretching velocity } U_{\mathrm{w}}(x) / U_{0}, \\
m>+1 & : \text { rapidly increasing stretching velocity } U_{\mathrm{w}}(x) / U_{0} .
\end{array}
$$

As it is well known, the problem (1)-(3) admits similarity solutions for all $-\infty<m<+\infty$ when the suction/injection velocity $V_{\mathrm{w}}(x)$ is proportional to $x^{(m-1) / 2}$. When $m \neq-1$, the components of the velocity field can be written in the form $[9,10]$,

$$
\begin{aligned}
& u(x, y)=U_{\mathrm{w}}(x) f^{\prime}(\eta), \quad \eta=\sqrt{\frac{\left|(m+1) x U_{\mathrm{w}}(x)\right|}{2 v}} \frac{y}{x}, \\
& v(x, y)=-s \sqrt{\frac{v\left|(m+1) U_{\mathrm{w}}(x)\right|}{2 x}}\left[f(\eta)+\frac{m-1}{m+1} \eta f^{\prime}(\eta)\right]
\end{aligned}
$$

where the dimensionless stream function $f=f(\eta)$ is obtained as solution of the two-point boundary value problem

$$
\begin{aligned}
& s f^{\prime \prime \prime}+f f^{\prime \prime}-\beta f^{\prime 2}=0, \\
& f(0)=f_{\mathrm{w}}, \quad f^{\prime}(0)=1, \quad f^{\prime}(\infty)=0 .
\end{aligned}
$$

In the above equations the prime denotes differentiations with respect to the similarity variable $\eta, f_{\mathrm{w}}$ is the mass transfer parameter (or, the dimensionless suction/injection velocity), $\beta \equiv 2 m /(m+1)$ and $s$ stands for the sign

$$
s=\operatorname{sgn}\left(U_{0}\right) \operatorname{sgn}(m+1) .
$$


The dimensional suction/injection velocity $v(x, 0)=V_{\mathrm{w}}(x)$ and the dimensional entrainment velocity $v(x, \infty)=V_{\infty}(x)$ are

$$
\begin{aligned}
& V_{\mathrm{w}}(x)=V_{0} \cdot\left(\frac{x}{L}\right)^{\frac{m-1}{2}}, \quad V_{0}=-s \sqrt{\frac{v\left|(m+1) U_{0}\right|}{2}} f_{\mathrm{w}}, \\
& V_{\infty}(x)=V_{\infty} \cdot\left(\frac{x}{L}\right)^{\frac{m-1}{2}}, \quad V_{\infty}=-s \sqrt{\frac{v\left|(m+1) U_{0}\right|}{2}} f_{\infty}
\end{aligned}
$$

where $f_{\infty} \equiv f(\infty)$ denotes the dimensionless entrainment velocity.

As shown in [11], the case of inverse linear stretching velocity $U_{\mathrm{W}}(x)=U_{0} L / x$ corresponding to $m=-1$ requires a special approach and will not further be considered here. We throughout assume in this paper that $m \neq-1$.

The scale factor $U_{0}$ of the stretching velocity is positive when the surface issues from the slot and moves toward $+\infty$, and negative when the surface moves from $+\infty$ toward 0 , where it enters the slot. These motions induce in the fluid two basically different velocity boundary layers, which, according to the nomenclature introduced by Goldstein [12], will be called forward (or usual) and backward boundary layers, respectively. The main difference between these types of boundary layers originates from the fact that in the former case the slot plays the role of a well definite leading edge, while in the latter one the leading edge recedes to an indefinite station far upstream. As a consequence, in the backward boundary layer the fluid loses any memory of the perturbation introduced by this indefinite leading edge. In this case, the slot at $x=0$ plays the role of a trailing edge. This is the reason why the forward and backward boundary layer flows corresponding, respectively, to $\operatorname{sgn}\left(U_{0}\right)=+1$ and $\operatorname{sgn}\left(U_{0}\right)=-1$, represent quite distinct physical phenomena. In the industrial manufacturing processes, both of these two types of boundary layers are of engineering interest. Forward boundary layers are generated in the ambient fluid, e.g., during the drawing of plastic sheets from a molten material, while, as described by Kuiken [8], during the cooling of a low-heat-resistance sheet, e.g., backward free convection boundary layers may occur.

A simple inspection of the basic differential equation (6) as well as of the various stretching regimes specified by the scheme (4) show that the four different flow regimes associated with the four possible combinations of $\operatorname{sgn}\left(U_{0}\right)= \pm 1$ and $\operatorname{sgn}(m+1)= \pm 1$ lead to two basically different boundary value problems (6), (7), corresponding to $s=+1$ and $s=-1$, respectively. To the best of our knowledge, in the literature no comprehensive investigation of all these forward and backward boundary layers exists. Even in the seminal paper of [9], only the impermeable case $f_{\mathrm{w}}=0$ with $s=+1$ for the forward boundary layers $\left(\operatorname{sgn}\left(U_{0}\right)=+1\right)$ in the range $m>-1$, and the backward ones $\left(\operatorname{sgn}\left(U_{0}\right)=-1\right)$ in the range $m<-1$ have been investigated. In [13] the case $s=-1$ for the forward boundary layers in the range $m<-1$ of the rapidly decreasing stretching velocities was considered, and in [11] results for the forward boundary layers for inverse linear stretching velocity, $m=-1$, were reported.

In the present paper we first consider both the forward and backward boundary layers, $\operatorname{sgn}\left(U_{0}\right)= \pm 1$, in the whole range $-\infty<m<+\infty$, except for $m=-1$. Our aim is to investigate a special physical and mathematical feature of the problem (6), (7), namely the relationship between the dimensionless wall shear stress

$$
f^{\prime \prime}(0) \equiv-S
$$

and the dimensionless entrainment velocity $f_{\infty}=f_{\infty}\left(m ; f_{\mathrm{W}}\right)$ in the parameter plane $\left(m, f_{\mathrm{W}}\right)$ specified by the stretching exponent $m$ and the mass transfer parameter $f_{\mathrm{W}}$. As we are aware, this problem has not yet been examined in detail. Concerning the effect of $f_{\mathrm{w}}$ on $S=S\left(m ; f_{\mathrm{w}}\right)$ and $f_{\infty}=f_{\infty}\left(m ; f_{\mathrm{w}}\right)$, the following theorem will be proven below.

At the value $f_{\mathrm{w}}=f_{\mathrm{w}}^{*}$ of the mass transfer parameter where the wall shear stress $S$ vanishes, the first derivative of the dimensionless entrainment velocity $f_{\infty}$ with respect to $f_{\mathrm{w}}$ also vanishes, and conversely.

This theorem, which builds a bridge between two physical quantities at the outer and inner edge of wall driven boundary layers, will be referred to for short as entrainment theorem, and it is the main finding of the paper. Its physical content will be analyzed in Sect. 4 in some detail. 


\section{Analytical solutions of the problem (6), (7)}

3.1 The special cases $m=-1 / 3$ and $m=-1 / 2$

As it is well known [10], in the special cases $m=-1 / 3$ and $-1 / 2$ the solution of the boundary value problem (6), (7) can be given for forward boundary layers, $\operatorname{sgn}\left(U_{0}\right)=+1$, in terms of elementary transcendental functions.

Case $m=-1 / 3$

$$
\begin{aligned}
& S=f_{\mathrm{w}}, \quad-\infty<f_{\mathrm{w}}<+\infty \\
& f_{\infty}=\sqrt{f_{\mathrm{w}}^{2}+2}=\sqrt{S^{2}+2}, \\
& f(\eta)=f_{\infty} \tanh \left(\frac{1}{2} f_{\infty} \eta+\ln \sqrt{\frac{f_{\infty}+f_{\mathrm{w}}}{f_{\infty}-f_{\mathrm{w}}}}\right),
\end{aligned}
$$

Case $m=-1 / 2$

$$
\begin{aligned}
& S=\frac{2 f_{\mathrm{w}}^{2}-1}{2 f_{\mathrm{w}}}, \quad 0<f_{\mathrm{w}}<+\infty, \\
& f_{\infty}=\left(f_{\mathrm{w}}^{3}+3 f_{\mathrm{w}}+\frac{9}{4 f_{\mathrm{w}}}\right)^{1 / 3} .
\end{aligned}
$$

The solution for the dimensionless stream function $f=f(\eta)$ can be obtained in this case only in the implicit form

$$
\eta=\frac{1}{f_{\infty}} \cdot \ln \left[\frac{F\left(W_{0}\right)}{F(W)}\right]
$$

where

$$
\begin{aligned}
& W=\sqrt{\frac{f}{f_{\infty}}}, \quad W_{0}=\sqrt{\frac{f_{\mathrm{w}}}{f_{\infty}}}, \\
& F(W)=(1-W)\left(1+W+W^{2}\right)^{-1 / 2} \exp \left[-3^{1 / 2} \cdot \arctan \left(\frac{1+2 W}{3^{1 / 2}}\right)\right] .
\end{aligned}
$$

It is worth emphasizing here that while the solution (11) exists for all values $-\infty<f_{\mathrm{w}}<+\infty$ of the mass transfer parameter, the implicit solution $\eta=\eta(f)$ corresponding to $m=-1 / 2$ does only exist when a lateral suction $\left(0<f_{\mathrm{w}}<+\infty\right)$ is applied [10].

\subsection{The Merkin transformation}

To prove the entrainment theorem, an extension of the Merkin transformation approach [14] to the present boundary value problem (6), (7) will be applied.

The basic feature of the Merkin transformation is that it reverses the role of the stream function $f$ in the boundary value problem (6), (7) from that of the old dependent variable to that of a new independent variable $\phi \equiv f_{\infty}-f$ and at the same time, it transfers the role of the dependent variable from $f$ to $p(\phi) \equiv \mathrm{d} f / \mathrm{d} \eta$. The main advantage of this order-lowering transformation is that it enables the calculation of the wall shear stress $S=S\left(m ; f_{\mathrm{w}}\right)$ and the entrainment velocity $f_{\infty}=f_{\infty}\left(m ; f_{\mathrm{w}}\right)$ without needing to know the solution $f=f\left(\eta ; m, f_{\mathrm{w}}\right)$ of the boundary value problem (6), (7), neither in an explicit nor in an implicit form.

First, we extend the Merkin transformation method [14] developed originally for an impermeable surface $f_{\mathrm{w}}=0$ and $s=+1$ to the cases $s= \pm 1$ and $f_{\mathrm{w}} \neq 0$ in which a lateral mass flux is present. To this end, we modify the transformation slightly by changing to a new independent variable $z$ and to a new dependent one, $Y=Y(z)$, which we define as follows:

$$
z=1-\frac{f}{f_{\infty}}, \quad Y=\frac{1}{f_{\infty}^{2}} \frac{\mathrm{d} f}{\mathrm{~d} \eta}
$$


Thus, the third-order boundary value problem (6), (7) reduces to the second-order one

$$
\begin{aligned}
& s \frac{\mathrm{d}}{\mathrm{d} z}\left(Y \frac{\mathrm{d} Y}{\mathrm{~d} z}\right)+(z-1) \frac{\mathrm{d} Y}{\mathrm{~d} z}-\beta Y=0, \\
& Y(0)=0, \quad Y\left(z_{0}\right)=\frac{1}{f_{\infty}^{2}}
\end{aligned}
$$

where

$$
z_{0}=\left.z\right|_{\eta=0}=1-\frac{f_{\mathrm{w}}}{f_{\infty}}
$$

The first condition (17) has been obtained from $f^{\prime}(\infty)=0$, and the second one from $f(0)=f_{\mathrm{w}}$ and $f^{\prime}(0)=1$. The wall shear stress $S$ is obtained in this approach as

$$
S=\left.f_{\infty} \frac{\mathrm{d} Y}{\mathrm{~d} z}\right|_{z=z_{0}}
$$

After the solution $Y=Y(z)$ of the transformed boundary value problem (16), (17) has been found, the solution $f=f(\eta)$ of the original problem (6), (7) can be obtained in the implicit form $\eta=\eta(f)$ by quadratures,

$$
\eta=-\frac{1}{f_{\infty}} \int_{z_{0}}^{1-\frac{f}{f \infty}} \frac{\mathrm{d} z}{Y(z)}
$$

\subsection{The series solution}

Looking for the solution of the boundary value problem (16), (17) in the power series form

$$
Y=\sum_{n=0}^{\infty} A_{n} z^{n}
$$

one obtains for the coefficients $A_{n}$ the system of equations (see also [15])

$s \sum_{n=0}^{k}(n+1)\left[(n+2) A_{n+2} A_{k-n}+(k-n+1) A_{n+1} A_{k-n+1}\right]=(k+1) A_{k+1}+(\beta-k) A_{k}, \quad k=0,1,2, \ldots$

The boundary condition $Y(0)=0$ implies $A_{0}=0$. Thus, one obtains from Eq. (22) for the next two coefficients the expressions

$$
A_{1}=s, \quad A_{2}=\frac{s}{4}(\beta-1) .
$$

The subsequent coefficients $A_{3}, A_{4}, A_{5}, \ldots$ can then be obtained recursively according to

$$
A_{k}=\frac{\beta-k+1}{k^{2}} A_{k-1}-s \frac{k+1}{2 k} \cdot \sum_{n=2}^{k-1} A_{n} A_{k-n+1}, k=3,4,5, \ldots
$$

Specifically,

$$
\begin{aligned}
& A_{3}=\frac{s}{72}\left(1-\beta^{2}\right), \\
& A_{4}=\frac{s}{576}\left(1-\beta^{2}\right)(1-2 \beta), \\
& A_{5}=\frac{s}{86400}\left(1-\beta^{2}\right)\left(11-81 \beta+88 \beta^{2}\right), \\
& A_{6}=\frac{s}{1036800}\left(1-\beta^{2}\right)\left(-9-125 \beta+447 \beta^{2}-337 \beta^{3}\right) .
\end{aligned}
$$


Then, the second boundary condition (17) yields for $f_{\infty}$ the equation

$$
f_{\infty}^{2} \sum_{k=1}^{\infty} A_{k}\left(1-\frac{f_{\mathrm{w}}}{f_{\infty}}\right)^{k}-1=0
$$

For the wall shear stress $S=S\left(m ; f_{\mathrm{w}}\right)$ one obtains from Eq. (19) the expression

$$
S=f_{\infty} \sum_{k=1}^{\infty} k A_{k}\left(1-\frac{f_{\mathrm{w}}}{f_{\infty}}\right)^{k-1}
$$

Equations (26) and (27) will play a key role in proving the entrainment theorem (see Sect. 4.1 below).

It is worth mentioning here that to the advantages of the Merkin transformation method belongs also the circumstance that the recurrence Eq. (24) allows for a rapid numerical and symbolical evaluation of the coefficients $A_{n}$ for any given value of $\beta$. In general, the absolute values of the coefficients $A_{k}$ decrease with increasing $k$ quite rapidly, except for the neighborhood of $\beta=-2$ (i.e., $\lambda=-1 / 2$ ). For instance, the terms of the sequence $\left|A_{k}\right|$ become smaller than $10^{-6}$ already after its 8 th term for $\beta=0$ (remember that $\left|A_{1}\right|=1$ for any $\beta$ ), after its 20 th term for $\beta=2$, after its 27 th term for $\beta=-1.5$, but for $\beta=-1.9$ they do only so after the 110th term of the series (21). The rate of convergence of the series occurring in Eqs. (26) and (27) can be accelerated with the aid of the classical Euler-Knopp type series transformation [16]. In the present calculations, following the work of [17], an improved form of the Euler-Knopp transformation has been used. Applied, e.g., to the series (21), the transformation of Gabutti and Lyness gives

$$
Y(z)=\sum_{n=0}^{\infty} \frac{n !}{(1-\varepsilon)^{n+1}}\left(\sum_{j=0}^{n} \frac{(-\varepsilon)^{n-j}}{(n-j) ! j !} A_{j} z^{j}\right) .
$$

Here $\varepsilon$ is a tuning parameter which can be chosen at convenience (for $\varepsilon=-1$ one recovers the classical Euler-Knopp type transformation). The other series Eqs. (26) and (27) can be transformed similarly. In order to check the convergence of the initial and of the transformed series for specified values of the parameters involved, it is useful to plot their respective terms in the increasing order of the summation indices. This procedure provides at the same time information about the number of terms which has to be considered for a required accuracy of the results [15].

\section{Discussion}

\subsection{The proof}

A straightforward analytical proof of the entrainment theorem can be given with the aid of Eqs. (26) and (27). Indeed, differentiating Eq. (26) once with respect to $f_{\mathrm{w}}$ one obtains

$$
2 f_{\infty} \frac{\mathrm{d} f_{\infty}}{\mathrm{d} f_{\mathrm{w}}} \sum_{k=1}^{\infty} A_{k}\left(1-\frac{f_{\mathrm{w}}}{f_{\infty}}\right)^{k}-\left(f_{\infty}-f_{\mathrm{w}} \frac{\mathrm{d} f_{\infty}}{\mathrm{d} f_{\mathrm{w}}}\right) \sum_{k=1}^{\infty} k A_{k}\left(1-\frac{f_{\mathrm{w}}}{f_{\infty}}\right)^{k-1}=0
$$

Solving this equation with respect to $\mathrm{d} f_{\infty} / \mathrm{d} f_{\mathrm{w}}$ and taking into account Eqs. (26) and (27) again, one obtains the relationship

$$
\frac{\mathrm{d} f_{\infty}}{\mathrm{d} f_{\mathrm{w}}}=\frac{f_{\infty} S}{f_{\mathrm{w}} S+2} .
$$

Now, Eq. (30) shows at the first glance that, when at some value $f_{\mathrm{w}}=f_{\mathrm{w}}^{*}$ of the mass transfer parameter the wall shear stress $S=S\left(m ; f_{\mathrm{w}}\right)$ possesses (for a specified $m$ ) a zero, then, $f_{\mathrm{w}}=f_{\mathrm{w}}^{*}$ yields at the same time a zero of the derivative $\mathrm{d} f_{\infty} / \mathrm{d} f_{\mathrm{w}}$ of the entrainment velocity and conversely, in full agreement with the statement of the entrainment theorem. It is an elementary exercise to show that in the case of the special solutions (11) and (12) the derivative $\mathrm{d} f_{\infty} / \mathrm{d} f_{\mathrm{w}}$ can also be put into the (generally valid) form (30). 


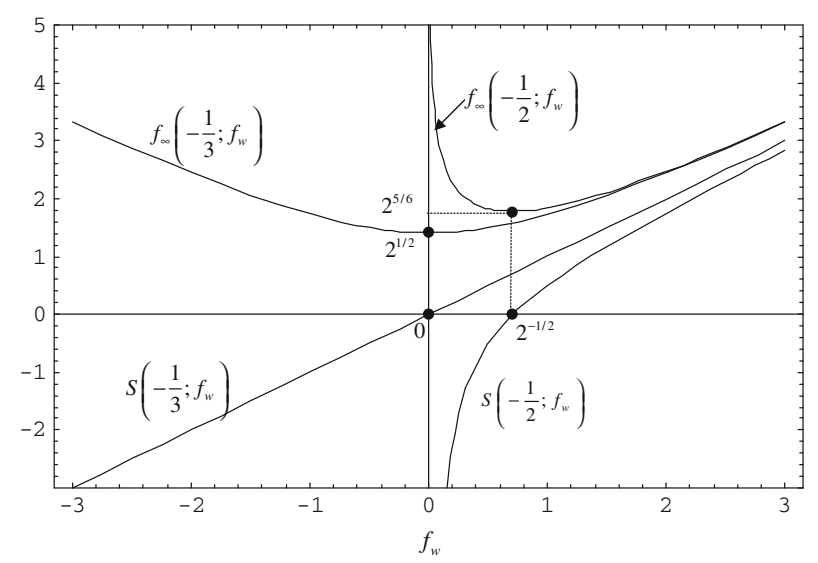

Fig. 1 Plots of the dimensionless entrainment velocity $f_{\infty}=f_{\infty}\left(m ; f_{\mathrm{w}}\right)$ and the wall shear stress $S=S\left(m ; f_{\mathrm{w}}\right)$ as functions of the mass transfer parameter $f_{\mathrm{w}}$ for $m=-1 / 3$ and $-1 / 2$, respectively. At $f_{\mathrm{w}}=f_{\mathrm{w}}^{*}$, where $f_{\infty}=f_{\infty}\left(m ; f_{\mathrm{w}}\right)$ reaches its (absolute) minimum, the wall shear stress becomes zero and changes sign, in full agreement with the entrainment theorem

Table 1 Validity ranges of the entrainment theorem

\begin{tabular}{lllll}
\hline$m$ & $s$ & Boundary layer & $f_{\mathrm{w}}$ & $f_{\infty}$ \\
\hline$m<-1$ & -1 & Forward $\left(U_{0}>0\right)$ & Suction: $f_{\mathrm{w}}<0$ & $f_{\infty}<0$ \\
$-1<m<0$ & +1 & Forward $\left(U_{0}>0\right)$ & Injection: $f_{\mathrm{w}}>0$ & $f_{\infty}>0$ \\
$m>0$ & -1 & Backward $\left(U_{0}<0\right)$ & Injection: $f_{\mathrm{w}}<0$ & $f_{\infty}<0$ \\
& & & Suction: $f_{\mathrm{w}}<0$ & Injection: $f_{\mathrm{w}}>0$ \\
\hline
\end{tabular}

\subsection{Validity range of the entrainment theorem}

The special solutions given in Sect. 3.1 offer a good opportunity for a simple preliminary validation of the entrainment theorem.

In the case $m=-1 / 3$, the statement of this theorem becomes evident by a simple inspection of Eqs. (11). Indeed, according to the first Eq. (11), the wall shear stress $S$ becomes zero for $f_{\mathrm{w}} \equiv f_{\mathrm{w}}^{*}=0$ (impermeable surface), where, according to the second Eq. (11), the entrainment velocity $f_{\infty}$ actually reaches its minimum value, $f_{\infty, \min }=\sqrt{2}$. In the case $m=-1 / 2$, elementary calculations show that both the wall shear stress $S$ and the first derivative of $f_{\infty}$ with respect to $f_{\mathrm{w}}, \mathrm{d} f_{\infty} / \mathrm{d} f_{\mathrm{w}}=\left(2 f_{\mathrm{w}}^{2}-1\right)\left(2 f_{\mathrm{w}}^{2}+3\right) /\left(2 f_{\mathrm{w}} f_{\infty}\right)^{2}$ become zero for $f_{\mathrm{w}}=1 / \sqrt{2}$. Thus, $f_{\mathrm{w}}^{*}=1 / \sqrt{2}$, and the corresponding minimum value of the entrainment velocity is $f_{\infty, \min }=2^{5 / 6}=1.781797$. These two particular cases of the entrainment theorem are illustrated in Fig. 1 where the respective quantities $f_{\infty}=f_{\infty}\left(m ; f_{\mathrm{w}}\right)$ and $S=S\left(m ; f_{\mathrm{w}}\right)$ have been plotted as functions of $f_{\mathrm{w}}$. One sees that $f_{\infty}\left(m ; f_{\mathrm{w}}^{*}\right)$, with $f_{\mathrm{w}}^{*}=0$ for $m=-1 / 3$, and $f_{\mathrm{w}}^{*}=1 / \sqrt{2}$ for $m=-1 / 2$ are absolute minima of the respective entrainment velocities, and at the same time $S\left(m ; f_{\mathrm{w}}^{*}\right)=0$, in full agreement with the entrainment theorem.

For other values of the stretching exponent $m$, it is obvious from the very beginning that the entrainment theorem can only become effective when, for a specified $s(s=+1$ or -1$)$, the boundary value problem (6), (7) admits solutions for vanishing value of the wall shear stress, $S=0$. In this respect, a heuristic analysis of the problem (6), (7) based on a point-mechanical analogy leads (similarly to procedure applied in $[11,18,19]$ ) to the results summarized in Table 1 below.

In Figs. 2, 3, 4 the correlation between the zeros of the wall shear stress $S=S\left(m ; f_{\mathrm{w}}\right)$ and the maxima/ minima of the dimensionless entrainment velocity $f_{\infty}=f_{\infty}\left(m ; f_{\mathrm{W}}\right)$ is illustrated for some selected values of the power law exponent $m$ in the ranges specified in Table 1. All these Figures confirm the statement of the entrainment theorem again. The special cases $m=-1 / 3$ and $-1 / 2$ shown in Fig. 1 belong to the $m$-range of the second row, $-1<m<0$, of Table 1 (forward boundary layers, $U_{0}>0, s=+1$ ). For more transparency, the cases of Fig. 1 have been included in Fig. 3 again. The values $f_{\mathrm{w}}^{*}$ of the mass transfer parameter $f_{\mathrm{w}}$ and of the corresponding extrema $f_{\infty, \text { extr }}=f_{\infty}\left(m ; f_{\mathrm{w}}^{*}\right)$ of the dimensionless entrainment velocity $f_{\infty}\left(m ; f_{\mathrm{w}}\right)$ are 


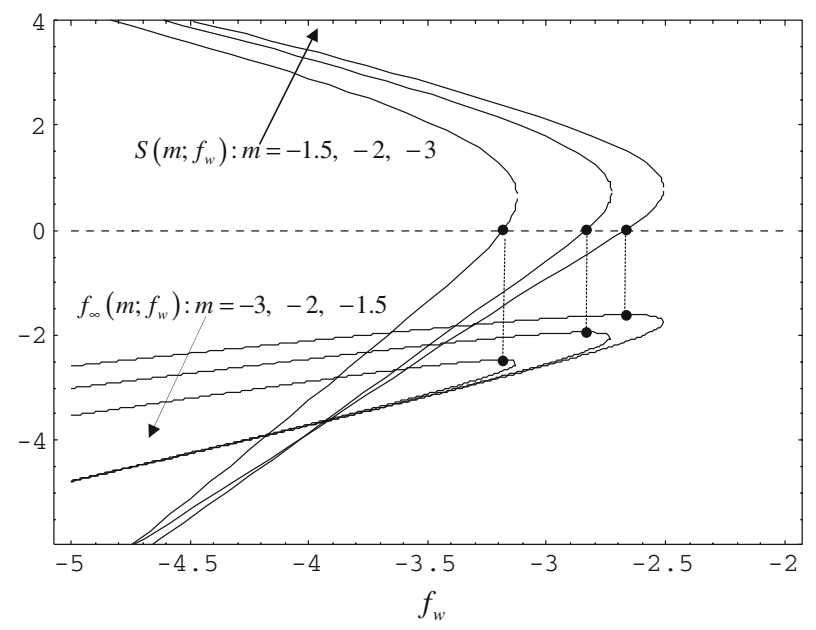

Fig. 2 Illustration of the entrainment theorem for three selected values of the power-law exponent in the range $m<-1$ (forward boundary layers, $U_{0}>0, s=-1$, see Tables 1,2$)$. The dots mark the absolute maxima of the entrainment velocities $f_{\infty}=$ $f_{\infty}\left(m ; f_{\mathrm{W}}\right)$ (curves in the lower half-plane), associated according to the entrainment theorem with zeros of the corresponding wall shear stresses $S=S\left(m ; f_{\mathrm{w}}\right)$

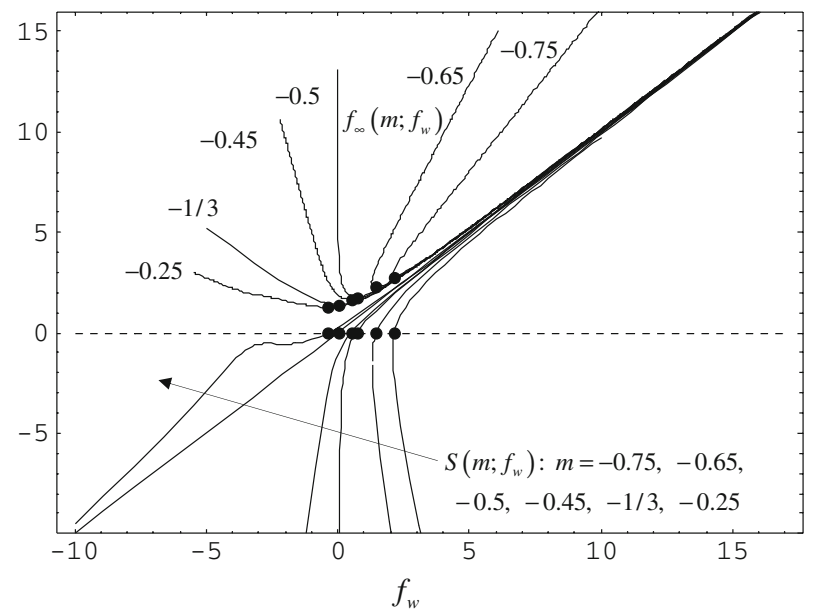

Fig. 3 Illustration of the entrainment theorem for six selected values of the power-law exponent in the range $-1<m<0$ (forward boundary layers, $U_{0}>0, s=+1$, see Tables 1,2$)$. The dots mark the absolute minima of the entrainment velocities $f_{\infty}=$ $f_{\infty}\left(m ; f_{\mathrm{W}}\right)$ (curves in the upper half-plane), associated according to the entrainment theorem with zeros of the corresponding wall shear stresses $S=S\left(m ; f_{\mathrm{w}}\right)$

collected in Table 2 for all the $m$ values selected for the Figs. 1, 2, 3, 4. The families of entrainment velocity curves $f_{\infty}=f_{\infty}\left(m ; f_{\mathrm{w}}\right)$ shown in Figs. 1, 2, 3, 4 represent the contour plots (topographic maps) of Eq. (26) for the specified values of $m$. The family of the corresponding wall shear stress curves $S=S\left(m ; f_{\mathrm{w}}\right)$ has then be obtained by substituting the explicit (numerical) solution of Eq. (26) into Eq. (27), and plotting the resulting expressions of $S$ as functions of the mass transfer parameter $f_{\mathrm{w}}$. There actually turns out that, for all specified values of $m$, the extrema $f_{\infty, \text { extr }}=f_{\infty}\left(m ; f_{\mathrm{w}}^{*}\right)$ of the entrainment velocity on the one hand, and the vanishing values $S\left(m ; f_{\mathrm{w}}^{*}\right)$ of the wall shear stress on the other hand, are reached at the same values $f_{\mathrm{w}}^{*}(m)$ of the mass transfer parameter $f_{\mathrm{w}}$, in full agreement with the entrainment theorem. In the range $m>0$ of Fig. 4 , the absolute maximum of the entrainment velocity $f_{\infty \text {. } \max }=f_{\infty}\left(m ; f_{\mathrm{w}}^{*}\right)$ goes to zero when $m \rightarrow 1$, and it is reached for $f_{\mathrm{w}}^{*} \rightarrow-\infty$. The corresponding backward boundary layer solution is available in an explicit analytical form [20], and yields for $f_{\infty}$ and $S$ the expressions

$$
f_{\infty}=\frac{1}{2}\left(f_{\mathrm{w}}+\sqrt{f_{\mathrm{w}}^{2}-4}\right), \quad S=-f_{\infty}, \quad f_{\mathrm{w}} \leq-2
$$




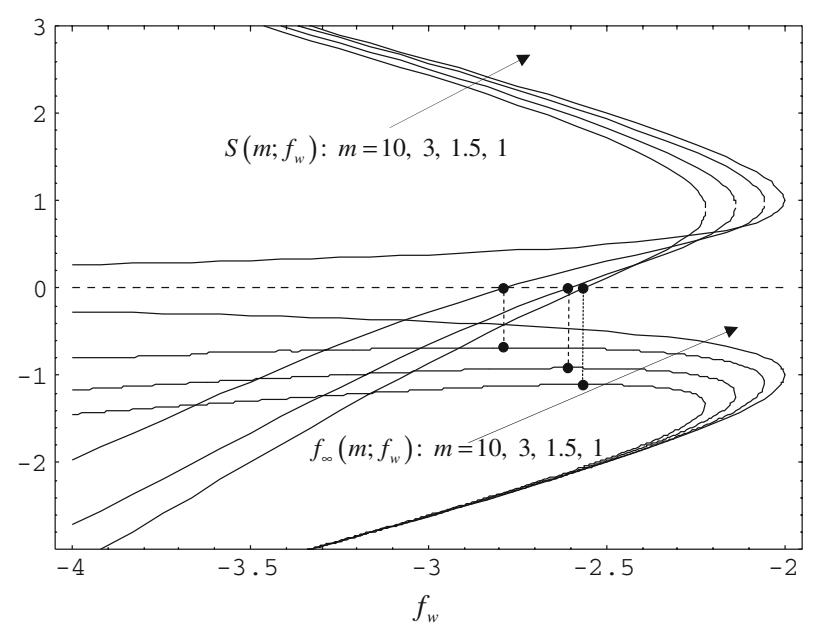

Fig. 4 Illustration of the entrainment theorem for three selected values of the power-law exponent in the range $m>0$ (backward boundary layers, $U_{0}<0, s=+1$, see Tables 1,2$)$. The dots mark the absolute maxima of the entrainment velocities $f_{\infty}=$ $f_{\infty}\left(m ; f_{\mathrm{w}}\right)$ (curves in the lower half-plane), associated according to the entrainment theorem with zeros of the corresponding wall shear stresses $S=S\left(m ; f_{\mathrm{w}}\right)$. In the case $m=1, f_{\infty, \max }=0$ and $f_{\mathrm{w}}^{*}=-\infty$

Table 2 Values $f_{\mathrm{w}}^{*}$ of the mass transfer parameter $f_{\mathrm{w}}$ and of the corresponding extrema $f_{\infty, \text { extr }}=f_{\infty}\left(m ; f_{\mathrm{w}}^{*}\right)$ of the dimensionless entrainment velocity $f_{\infty}\left(m ; f_{\mathrm{w}}\right)$, for the $m$ values selected in Figs. 1, 2, 3, 4

\begin{tabular}{llll}
\hline$m$ & $f_{\mathrm{w}}^{*}$ & $f_{\infty, \text { extr }}$ & Comment \\
\hline-3 & -2.66878 & -1.61727 & Absolute maxima of $f_{\infty}$, Fig. 2 \\
-2 & -2.83431 & -1.94555 & \\
-1.5 & -3.18326 & -2.47355 & Absolute minima of $f_{\infty}$, Figs. 1,3 \\
-0.75 & 2.14190 & 2.80044 & \\
-0.65 & 1.45465 & 2.27539 & \\
$-1 / 2$ & $2^{-1 / 2}=0.70711$ & $2^{5 / 6}=1.78180$ & \\
-0.45 & 0.49043 & 1.65792 & \\
$-1 / 3$ & 0 & $\sqrt{2}=1.41421$ & Absolute maxima of $f_{\infty}$, Fig. 4 \\
-0.25 & -0.36667 & 1.26664 & \\
+1 & $-\infty$ & 0 & \\
+1.5 & -2.78856 & -0.67845 & \\
+3 & -2.60892 & -0.91601 & \\
+10 & -2.56337 & -1.10569 & \\
\hline
\end{tabular}

These equations show clearly that for $f_{\mathrm{w}} \rightarrow-\infty, f_{\infty} \rightarrow f_{\infty, \max }=0$ and $S \rightarrow 0$ as mentioned above, and are in full agreement with the entrainment theorem. These limiting features (i.e., $f_{\infty} \rightarrow f_{\infty, \max }=0$ and $S \rightarrow 0$ when $f_{\mathrm{w}} \rightarrow-\infty$ ) still persist in the whole interval $0<m \leq 1$, while finite values of $f_{\infty, \max }$ only for $m>1$ occur (see Fig. 4, Table 2).

It is worth mentioning here that, in addition to the entrainment theorem, Figs. 2, 3, 4 also illustrate the abundant occurrence of dual solutions associated with different values of $S$, for specified values of the parameters $m$ and $f_{\mathrm{w}}$. Dual and multiple solutions are frequently encountered in the boundary layer theory. In the prominent case of the mixed convection over a horizontal plate, e.g., this feature has first been reported by Schneider [21]. Professor Schneider's seminal work [21] has also inspired several subsequent papers concerned with various specific aspects and extensions of this basic phenomenon [22-26].

The physical origin of the entrainment theorem has to be sought in the sign change of the wall shear stress. This phenomenon is illustrated in Fig. 5, where the dimensionless velocity profiles corresponding to five selected values of the mass transfer parameter $f_{\mathrm{w}}$ have been plotted as functions of $\eta$ for $m=-1 / 2$. One of them (profile 3 ) corresponds to a vanishing value of the wall shear stress, $S=S\left(m ; f_{\mathrm{w}}^{*}\right)=0$, and to the smallest value of the entrainment velocity, $f_{\infty}=f_{\infty, \min }=f_{\infty}\left(m ; f_{\mathrm{w}}^{*}\right)=2^{5 / 6}=1.7818$, with $f_{\mathrm{w}}^{*}=1 / \sqrt{2}$. The other four profiles correspond to the indicated values of $f_{\mathrm{w}}$, below of $f_{\mathrm{w}}=f_{\mathrm{w}}^{*}=1 / \sqrt{2}$ (profiles 1 and 2) and above of $f_{\mathrm{w}}=f_{\mathrm{w}}^{*}=1 / \sqrt{2}$ (profiles 4 and 5) they are associated with larger values of $f_{\infty}$ than $f_{\infty, \min }$. Having in mind that in the case of profiles 1 and 2 the fluid velocity $f^{\prime}(\eta)$ is smaller than the wall velocity 


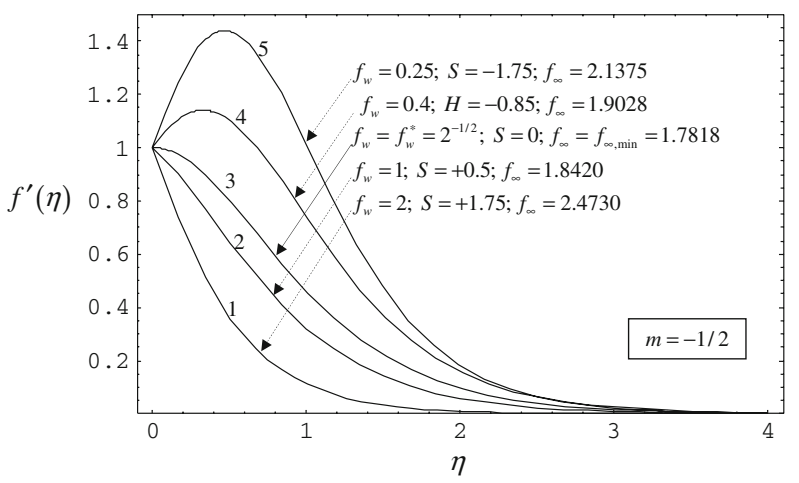

Fig. 5 Five dimensionless velocity profiles for $m=-1 / 2$. The middle one represents the profile with vanishing wall shear stress, $S=0$, and with the smallest entrainment velocity, $f_{\infty}=f_{\infty \text {, min }}$, and corresponds to $f_{\mathrm{w}}=f_{\mathrm{w}}^{*}=1 / \sqrt{2}$. The upper two profiles with $f_{\mathrm{w}}<f_{\mathrm{w}}^{*}$, are associated with negative values of $S$ and the lower ones with $f_{\mathrm{w}}>f_{\mathrm{w}}^{*}$, correspond to positive values of $S$

$f^{\prime}(0)=1$ at all stations $\eta>0$, the positive sign of wall shear stress, $S=-f^{\prime \prime}(0)>0$, corresponds to our physical expectation. It is also clear that in this case, the larger the value of the suction parameter $f_{\mathrm{w}}$, the larger the corresponding entrainment velocity $f_{\infty}$ and wall shear stress $S$. However, in the case of temperature profiles 4 and 5, it is not immediately clear why the wall shear stress has changed its sign from $S>0$ to $S<0$, and why the entrainment velocities become larger than $f_{\infty, \min }$, although in this case the respective values of the suction parameter $f_{\mathrm{w}}$ are smaller than $f_{\mathrm{w}}^{*}$.

Obviously, the reversed wall shear stresses (negative $S$ values) are related to the "velocity hills" of the profiles 4 and 5. But, where do these velocity hills come from? Having in mind that for $m<0$ the local wall velocity $U_{\mathrm{w}}(x) / U_{0}=(x / L)^{m}$ decreases with increasing $x$, the fluid accelerated upstream arrives to slowly moving portions of the wall at all the downstream stations. Consequently, for small values of the suction parameter $f_{\mathrm{w}}$ "velocity spots" may occur which in turn lead to a reversed wall shear stress and, at the same time, to larger values of the entrainment velocity than $f_{\infty, \min }$. The smaller the suction parameter the higher the velocity hills and the larger the corresponding entrainment velocities $f_{\infty}$ in the low suction range $0<f_{\mathrm{w}}<f_{\mathrm{w}}^{*}$. In the strong suction range $f_{\mathrm{w}}>f_{\mathrm{w}}^{*}$ of the profiles 1 and 2 on the other hand, the velocity hills are "sucked out" from the fluid and, consequently, "usual" wall shear stress and entrainment mechanisms become effective. A similar behavior occurs in the case of backward boundary layers in the range $m>1$.

\section{Summary and conclusions}

In the present paper the forward and backward boundary layer flows driven by permeable plane surfaces stretching with power-law velocities have been considered in the presence of an applied lateral mass flux. The relationship between the dimensionless entrainment velocity and the wall shear stress has been investigated in detail. The main result of the paper is summarized in the entrainment theorem which asserts that, at the value of the mass transfer parameter where the wall shear stress vanishes, the entrainment velocity reaches a minimum or maximum value. The entrainment theorem builds a bridge between two flow characteristics at the outer and inner edge of the wall driven boundary layer, respectively, and applies for all values of the stretching exponent $m$ (according to the specifications of Table 1). The converse of its statement is also true. The physical reason for this relationship resides in the velocity overshoot occurring for negative values of the wall temperature exponent $m$ in the case of the forward, and for positive $m^{\prime} s$ in the case of the backward boundary layers, respectively. We mention that in the physically different but mathematically identical context of the Darcy free convection over a vertical plate with power law temperature distribution a similar theorem holds.

\section{References}

1. Sakiadis, B.C.: Boundary-layer behavior on continuous solid surfaces. AIChE J. 7 (1961) 26-28. See also pp. 221-225, $467-472$ 
2. Pop, I., Ingham, D.B.: Convective Heat Transfer: Mathematical and Computational Modeling of Viscous Fluids and Porous Media. Pergamon, Oxford (2001)

3. Erickson, L.E., Fan, L.T., Fox, V.G.: Heat and mass transfer on a moving continuous flat plate with suction or injection. Ind. Eng. Chem. 5, 19-25 (1966)

4. Crane, L.E.: Flow past a stretching plane. J. Appl. Math. Phys. (ZAMP) 21, 645-647 (1970)

5. Vleggaar, J.: Laminar boundary layer behavior on continuous stretching surfaces. Chem. Eng. Sci. 32, 1517-1525 (1977)

6. Gupta, P.S., Gupta, A.S.: Heat and mass transfer on a stretching sheet with suction or blowing. Can. J. Chem. Eng. 55, 744-746 (1977)

7. Afzal, N., Varshney, I.S.: The cooling of a low heat resistance stretching sheet moving through a fluid. Wärme Stoffübertragung 14, 289-293 (1980)

8. Kuiken, H.K.: A backward free-convective boundary layer. Q. J. Mech. Appl. Math. 34, 397-413 (1980)

9. Banks, W.H.H.: Similarity solutions of the boundary layer equations for a stretching wall. J. Mécanique Theor. Appl. 2 , 375-392 (1983)

10. Magyari, E., Keller, B.: Exact solutions for self-similar boundary-layer flows induced by permeable stretching walls. Eur. J. Mech. B Fluids 19, 109-122 (2000)

11. Magyari, E., Pop, I., Keller, B.: The missing similarity boundary-layer flow over a moving plane surface. J. Appl. Math. Phys. (ZAMP) 53, 782-793 (2002)

12. Goldstein, S.: On backward boundary layers and flow in converging passages. J. Fluid Mech. 21, 33-45 (1965)

13. Magyari, E., Ali, M.E., Keller, B.: Heat and mass transfer characteristics of the self-similar boundary-layer flows induced by continuous surfaces stretched with rapidly decreasing velocities. Heat Mass Transf. 38, 65-74 (2001)

14. Merkin, J.H.: A note on the solution of a differential equation arising in boundary-layer theory. J. Eng. Math. 18, 31-36 (1984)

15. Magyari, E., Keller, B.: A direct method to calculate the heat transfer coefficient of steady similar boundary layer flows induced by continuous moving surfaces. Int. J. Thermal Sci. 44, 245-254 (2005)

16. Knopp, K.: Theory and Application of Infinite Series. Dover, New York (1990)

17. Gabutti, B., Lyness, J.N.: Some generalizations of the Euler-Knopp transformation. Numer. Math. 48, 199-220 (1986)

18. Magyari, E., Pop, I., Keller, B.: New similarity solutions for boundary layer flow on a horizontal surface in porous medium. Trans. Porous Media 51, 123-140 (2003)

19. Magyari, E., Keller, B.: Buoyancy sustained by viscous dissipation. Trans. Porous Media 53, 105-115 (2003)

20. Magyari, E., Keller, B.: Backward free convection boundary layers in porous media. Trans. Porous Media 55, 285-300 (2004)

21. Schneider, W.: A similarity solution for combined forced and free convection flow over a horizontal plate. Int. J. Heat Mass Transf. 22, 1401-1406 (1979)

22. Steinrück, H.: Mixed convection over a cooled plate. Nonuniqueness ad numerical instabilities of the boundary layer equations. J. Fluid Mech. 278, 251-265 (1994)

23. Steinrück, H.: Mixed convection over a horizontal plate: self-similar and connecting boundary layer flows. Fluid Dyn. Res. 15, 113-127 (1995)

24. Steinrück, H.: Mixed convection over a horizontal plate: connecting boundary layer flows. J. Appl. Math. Mech. (ZAMM) 76(S5), 503-504 (1996)

25. Steinrück, H.: A review of the mixed convection boundary-layer flow over a cooled horizontal plate. GAMM-Mitt. Heft 2 , 127-158 (2001)

26. Magyari, E., Pop, I., Keller, B.: Mixed convection boundary layer flow past a horizontal permeable plate. Fluid Dyn. Res. 31, 215-225 (2002) 